\title{
White Matter Hyperintensities on 1.5 and 3 Tesla Brain MRI in Healthy I ndividuals
}

\author{
Carol Di Perri ${ }^{1,2}$, Michael G. Dwyer ${ }^{1}$, Niels Bergsland ${ }^{1}$, Claudiu Schirda ${ }^{1}$, Guy U Poloni ${ }^{1}$, David \\ Wack $^{1}$, Jennifer L. Cox ${ }^{1}$, Turi O. Dalaker ${ }^{1,4,5}$, Laura Ranza ${ }^{2}$, Erik Saluste ${ }^{3}$, Sara Hussein ${ }^{1}$, \\ Stefano Bastianello ${ }^{2}$, Robert Zivadinov ${ }^{1}$
}

1. Buffalo Neuroimaging Analysis Center, The Jacobs Neurological Institute, Department of Neurology, State University of New York, Buffalo, NY, USA. 2. Department of Neuroradiology, IRCCS, C. Mondino, University of Pavia, Pavia, Italy. 3. Buffalo Niagara MRI Center, Buffalo General Hospital, Kaleida Health, Buffalo, NY, USA. 4. Department of Radiology, Stavanger University Hospital, Stavanger, Norway. 5. The Norwegian Centre for Movement Disorders, Stavanger University Hospital, Stavanger, Norway

Correspondence: Robert Zivadinov, MD, PhD. Address: Department of Neurology, School of Medicine and Biomedical Sciences, Buffalo Neuroimaging Analysis Center, 100 High St., Buffalo, NY 14203, USA. Email: rzivadinov@bnac.net.

Received: November 25, 2012

Accepted: December 18, 2012

Online Published: April 22, 2013

DOI : $10.5430 / j b g c . v 3 n 3 p 53$

URL: http://dx.doi.org/10.5430/jbgc.v3n3p53

\section{Abstract}

Background: White matter hyperintensities (WMH) on T2-weighted magnetic resonance imaging (MRI) are frequent incidental findings in brains of asymptomatic healthy individuals (HI). The morphological and spatial distribution of these WMH between standard 1.5T scanners and increasingly available 3T scanners in a large population of HI in clinical routine settings has not been investigated.

Objectives: To investigate the effect of changing from a 1.5T to a 3T MRI scanner on the number, volume and spatial distribution of WMH on brain MRI in a population of clinically HI.

Materials and Methods: Fifty-three (53) HI were examined using T2 weighted sequences on both 1.5T and 3T within one week in a random order. The WMH were outlined semi-automatically by two blinded operators. Number and volume of WMH were calculated. Spatial lesion distribution was assessed using WMH probability maps (WMHPM). Paired-wise analysis examined the proportion of WMH not found on 1.5T and/or 3T. A posteriori unblinded analysis was conducted to examine the non-overlapping identifications of WMH between the 1.5T and 3T.

Results: For paired-wise WMH analysis, 3T showed significantly higher WMH number and individual volume compared to $1.5 \mathrm{~T}(p<0.001)$. Logistic regression analysis showed that likelihood of missing WMH on 1.5T was significantly higher for smaller WMH. WMHPM revealed spatial WMH differences on 3T compared to 1.5T, with WMH more pronounced in the occipital regions with higher field strength.

Conclusion: This study showed that use of higher magnetic field strength identified more WMH in healthy subjects with respect to both morphological and spatial characteristics. These WMH do not necessarily represent pathology; however, these findings should be taken into account in lesion segmentation on a 3T MRI and might suggest the use of universally accepted guidelines for lesions' segmentation on higher magnetic field, especially when it is executed by different operators and/or centers. 


\section{Key words}

White matter hyperintensities, Field strength, Healthy controls, Voxel-wise comparison

\section{I ntroduction}

The widespread availability of magnetic resonance imaging (MRI) has resulted in an increased recognition of white matter hyperintensities (WMH) on T2 weighted image (WI) MRI scans as common incidental findings in brains of clinically healthy individuals (HI) ${ }^{[1-5]}$. Studies have found increased prevalence of WMH with an increasing age, and incidental WMH were seen as early as middle age and even in young adults ${ }^{[3,6]}$.

Research over the past decade has been somewhat controversial about the clinical impact of these WMH in the general population. It has been found that WMH are associated with physical disability, cognitive deficits ${ }^{[7,8]}$, gait abnormality ${ }^{[9]}$, and psychiatric disorders such as depression ${ }^{[10]}$.

As high-field MRI systems that offer superior image quality become increasingly available in clinical routine care ${ }^{[11,12]}$, it is important to understand to what extent the higher magnetic strength may influence the detection rate of WMH in clinically $\mathrm{HI}$ and whether it could provide new clinical and neuropathological meanings. Higher strength fields have shown to detect more T2 hyperintense lesions in comparison to lower strength field, in pathologies such as clinically defined MS ${ }^{[11-15]}$, clinically isolated syndrome ${ }^{[16]}$ partial epilepsy of uncertain focus ${ }^{[17]}$ and cerebral microbeeds ${ }^{[18]}$. Recently, an increased detection rate of WMH on 3T scans was preliminarily shown in a small sample of $\mathrm{HI}^{[19]}$.

Past studies have used voxel-wise techniques to compare WMH distributions across populations in statistical terms in different diseases ${ }^{[1,20-22]}$, and the resulting statistical (non) parametric maps and WMH probability maps (WMHPMs) have been shown to be powerful tools for studying in vivo T2 WMH distribution. To the best of our knowledge, the effect of changing from a 1.5T to a 3T MRI on the number, volume and spatial distribution of WMH on brain MRI in a large and broad age sample of clinically $\mathrm{HI}$ has not been previously investigated using WMH paired- and voxel-wise fullyautomated comparison procedures. We hypothesized that use of 3T MRI would be superior to $1.5 \mathrm{~T}$ in the detection of WMH.

\section{Methods}

\subsection{Study population}

Our study consisted of $53 \mathrm{HI}$. The subjects were included if they had a normal MRI health screening questionnaire, and normal standard medical and neurological examination. For a brief assessment of cognitive function we conducted screening with the Mini Mental State Examination (MMSE) ${ }^{[23]}$ and the Symbol Digit Modality Test (SDMT) ${ }^{[24]}$. Exclusion criteria included a history of neurological or psychiatric disorders (including traumatic brain injury with loss of consciousness > $5 \mathrm{~min}$ ), abnormal MMSE and SDMT exams, presence of vascular risk factors (cardiovascular and cerebrovascular) and history of alcohol abuse. The following vascular risk factors were considered: current smoking, hypertension, diabetes mellitus, hypercholesterolemia, angina/myocardial infarct, atrial fibrillation, TIA/stroke, peripheral vascular disease and use of medications for hypertension, diabetes mellitus, hypercholesterolemia and anxiety/depression. The study was approved by an internal Institutional Review Board and written informed consent was obtained from all participating subjects.

\subsection{MRI examination}

All subjects were examined on both 1.5T and 3T scanners within one week. The order in which subjects were scanned in the two machines was randomized. All scans were performed on a 3T GE Signa Excite HD 12.0 Twin Speed 8-channel 
scanner (General Electric, Milwaukee, WI) and on a 1.5T GE Signa Excite HD 12.0 Echospeed 8-channel scanner (General Electric, Milwaukee, WI). The maximum slew rate and maximum gradient amplitudes in each orthogonal plane for the $3 \mathrm{~T}$ scanner were $150 \mathrm{~T} / \mathrm{m} / \mathrm{s}$ and $50 \mathrm{mT} / \mathrm{m}$ (zoom mode), and for the $1.5 \mathrm{~T}$ scanner $120 \mathrm{~T} / \mathrm{m} / \mathrm{s}$ and $33 \mathrm{mT} / \mathrm{m}$. A multi-channel head and neck (HDNV) coil manufactured by GE (General Electric, Milwaukee, WI) on 3T and a multi-channel HDNV coil manufactured by Medrad (Medrad, Pittsburgh, PA) on 1.5T were used to acquire the following sequences: multi-planar dual fast spin-echo (FSE) proton density (PD) and T2-weighted image (WI), Fluid-Attenuated Inversion-Recovery (FLAIR) and spin echo (SE) T1-WI.

Due to hardware differences and different spin relaxation properties at the two field strengths, the sequences were not perfectly identical on the two scanners. Nevertheless, sequences were optimized as much as possible to obtain similar T1 and T2 contrast on both scanners. An important aspect of the study, potentially affecting the WMH detection between the two scanners, was the slightly higher spatial resolution used on the 3T compared to $1.5 \mathrm{~T}$ (approx. 15\% smaller voxels at 3T compared to 1.5T). As our primary goal was to determine the real-world impact of switching from 1.5T to 3T in clinical practice, we created imaging protocols that we believe were "optimal" at each field strength. While most sequences at 1.5T are limited to a $256 \times 192$ matrix by the low SNR in a clinical scanning set-up (limited scan time), our initial protocol development experiments showed that it would be beneficial to increase resolution when going to 3T in order to detect more WMH, as we believe that keeping the same resolution between the two scanners would not be the most efficient (or most common) way to increase WMH detection power on 3T. Nevertheless, in order to investigate how the differing spatial resolution between the two field strengths might have affected our study results, we scanned two volunteers on the 3T scanner using two different choices of acquisition matrices and FOVs (the matrix/FOV used for 3T acquisitions and the matrix/FOV used for 1.5T, the rest of the parameters remained the same). WMH measurements were conducted by two blinded neuroradiologists and negligible differences in their volumes were demonstrated. A summary of scanning parameters is shown in Table 1.

Table 1. T1 and T2 MRI parameters at $1.5 \mathrm{~T}$ and 3T scanners

\begin{tabular}{|c|c|c|c|c|c|c|}
\hline & \multicolumn{2}{|l|}{ SE-T1 } & \multicolumn{2}{|l|}{ FLAIR } & \multicolumn{2}{|c|}{ FSE PD/T2-WI } \\
\hline & $3 T$ & $1.5 \mathrm{~T}$ & $3 T$ & $1.5 \mathrm{~T}$ & $3 T$ & $1.5 \mathrm{~T}$ \\
\hline Matrix & $256 \times 256$ & $256 \times 192$ & $256 \times 256$ & $256 \times 192$ & $256 \times 256$ & $256 \times 192$ \\
\hline $\mathrm{FOV}(\mathrm{cm})$ & 25.6 & 24 & 24 & 25.6 & 24 & \\
\hline $\mathrm{TE}(\mathrm{ms})$ & 9 & 12 & 120 & 120 & $12 / 95$ & $10 / 90$ \\
\hline $\mathrm{TR}(\mathrm{ms})$ & 600 & 450 & 8500 & 8000 & 3000 & 7475 \\
\hline ETL & 1 & 1 & 28 & 26 & 14 & 12 \\
\hline $\mathrm{AT}(\mathrm{min}, \mathrm{sec})$ & 4.07 & 6.40 & 4.16 & 5.21 & 4.31 & 4.07 \\
\hline Slices & 47 & 46 & 46 & 46 & 48 & 46 \\
\hline Thicks(mm) & 3 & 3 & 3 & 3 & 3 & 3 \\
\hline Gap & No & No & No & No & No & No \\
\hline Averages & 1 & 2 & 1 & 1 & 1 & 1 \\
\hline $\mathrm{TI}(\mathrm{ms})$ & - & - & 2100 & 2000 & - & - \\
\hline Flip & 90 & 60 & 90 & 90 & 90 & 90 \\
\hline Angle(degrees) & & & & & & \\
\hline Bandwidth(Hz/px) & 244 & 163 & 217 & 195 & 163 & 177 \\
\hline
\end{tabular}

Note. FOV: field of view; TE: echo time; TR: repetition time; ETL: echo train length; AT: acquisition time; TI: inversion time; FSE PD: fast spin-echo proton density; T2-WI: T2 weighted image; FLAIR: Fluid-Attenuated Inversion-Recovery; SE-T1-WI: spin echo T1-WI

An effort was made to maintain identical scanning conditions between both fields of strength, including use of the same head-supporting devices in order to match head position, as well as the same degree of inclination and table-head-magnet distance. The two scanners were located in the same building, on the same floor, across from one another, in identical humidity and temperature conditions. During the time of the study, monthly quality assurance sessions were carried out on the scanners and no major hardware upgrades were implemented. 


\subsection{MR data analysis}

\section{WMH volume analysis}

WMH were detected on FLAIR images, using PD/T2-WI and T1-WI to increase confidence in lesion detection. All WMH detection was performed independently on both $1.5 \mathrm{~T}$ and $3 \mathrm{~T}$ images by two operators blinded to scan identity and field strength. Discrepancies in WMH classification were resolved by mutual agreement. The Cohen's kappa statistics were obtained to quantify the level of intra- and inter-rater agreement between $1.5 \mathrm{~T}$ and 3T scanners. The inter-rater agreement was 0.808 (SD 0.01) for 1.5T and 0.807 (SD 0.02) for 3T, whereas the intra-rater agreement was 0.851 (SD 0.06) for 1.5T and $0.853(0.07)$ for $3 \mathrm{~T}$.

A highly reproducible semi-automated local thresholding technique for lesion segmentation, as previously described, was used for outlining of $\mathrm{WMH}^{[25]}$. The WMH were defined as FLAIR abnormal hyperintensities in normal appearing brain tissue, per experienced neuroradiologists; any artifact or normal findings such as septal hyperintensity, corticospinal tract hyperintensity and ventriculare CSF flow artifacts were carefully excluded.

We decided not to make a distinction between periventrivular WMH and deep WMH, as their possible different clinical and neuropathological impact has been largely questioned ${ }^{[20,26-28]}$.

Small caps and pencil-thin rims were not considered as WMH for this analysis. Periventricular "rims" and frontal and occipital "caps" were taken into the analysis only if extended ${ }^{[6]}$.

\section{Paired-wise WMH analysis}

To avoid biasing the results by resampling data from one scanner and not the other, both 1.5 and 3T images were resampled into a common halfway space on a subject-by-subject basis. To accomplish this, we used FSL's FLIRT utility ${ }^{[29]}$, allowing 6 degrees of freedom for the transformation.

For each subject, 3D clusters were formed from the union of the binary WMH masks. A table was formed relating each cluster to the corresponding values of WMH size (calculated as a sum of voxels times the voxels' size in $\mathrm{mm}^{3}$ ) for both the 1.5T and 3T scans. In the event that a cluster was created only from the mask of one of the scans, the WMH size of the "missing" WMH was reported as zero. The number of WMH found on the 3T scanner and not the 1.5T scanner was calculated by subtracting the number of clusters with a size of zero on the 1.5T scanner from the total number of clusters.

\section{Voxel-Wise Analysis (WMH probability map - WMHPM)}

To evaluate and quantify the spatial component of WMH differences between 1.5T and 3T scanners, we used a voxel-wise statistical non-parametric mapping technique similar to voxel-based morphometry ${ }^{[30]}$. First, we co-registered all FLAIR images to the Montreal Neurological Institute standard-space image (MNI152 template) using a 12-parameter affine model via FMRIB's FLIRT ${ }^{[29]}$ linear registration tool. The resulting transformation matrices were then applied to the corresponding WMH mask images to bring these into a standard space. All registrations were checked visually to exclude alignment failures. WMH mask images in standard space were then smoothed using a Gaussian kernel with a standard deviation of 4mm (equivalent to an FWHM of approximately 9.42mm).

These standard-space masks were then statistically compared on a voxel-wise basis using a mass-univariate permutation technique (FMRIB's randomise utility) ${ }^{[31]}$. The non-parametric equivalent of a paired t-test was performed at each voxel, with the pairs being within-subject $1.5 \mathrm{~T}$ and $3 \mathrm{~T}$ lesion masks. To correct for multiple comparisons while accounting for the underlying smoothness of the data, we employed the Threshold-Free Cluster Enhancement (TFCE) technique ${ }^{[32]}$. This approach is an alternative to standard cluster-based methods which suffer from the need for specification of an arbitrary cluster-forming threshold. From this analysis, we obtained a voxel-wise map of those areas differing significantly ( $p<$ 0.05 corrected) in the presence of WMH between 1.5 and 3T. 


\section{A posteriori unblinded analysis of non-concordant areas}

Additional analysis was conducted to examine the potential a-posteriori concordance of all a-priori non-concordant (non-overlapping) WMH between the 1.5T and 3T scanners. After excluding all overlapping WMH between the 1.5T and 3T scanners in the paired-wise analysis, the remaining WMH detected only on 1.5T and 3T were compared side by side in an unblinded manner. If recognized as concordant, the previously undetected WMH were contoured with full information from the other image. An inter-scanner comparison was then performed, calculating the number and percentage of these newly assigned $\mathrm{WMH}$.

\subsection{General statistics}

Statistical analysis was performed using the Statistical Package for the Social Science (SPSS, version 16.0, Chicago, IL). Values of MR measures (WMH volume, number and individual volume) were compared between 1.5T and 3T using the non-parametric Wilcoxon-Signed rank test or Mann-Whitney U-test, as appropriate. A Chi-Square test was used to compare the frequencies of WMH found in 1.5T to the ones in 3T. Data were considered significant at the $p<0.05$ level.

Pair-wise and a-posteriori non-concordant WMH analyses were performed using in-house software written in MatLab (The MathWorks, Inc., Natick, MA). Logistic regression was used to predict whether WMH would be missed on 1.5T, based on the individual volume of the WMH. Voxel-wise analysis was conducted directly on the images, as described above.

\section{Results}

\section{Demographic characteristics}

The study included 53 HC (sex: 36 females, 17 males; age in years: mean 39.4, median 39, range 18-63). The mean MMSE was 29.2 (SD 0.8) and the mean SDMT was 64.2 (SD 6.5).

\section{Total WMH volume per subject based differences between 1.5T and 3T}

Total WMH volume was significantly higher $(p<0.001)$ on 3T compared to $1.5 \mathrm{~T}$. Total WMH mean volume on $1.5 \mathrm{~T}$ was $155.3 \mathrm{~mm}^{3}$, (SD $190 \mathrm{~mm}^{3}$, median $68.5 \mathrm{~mm}^{3}$, $\min 0 \mathrm{~mm}^{3}$, max $752.6 \mathrm{~mm}^{3}$ ), whereas total WMH mean volume on 3T was $523.7 \mathrm{~mm}^{3}$, (SD $546.5 \mathrm{~mm}^{3}$, median $374.4 \mathrm{~mm}^{3}$, $\min 0 \mathrm{~mm}^{3}$, $\max 2460 \mathrm{~mm}^{3}$ ).

\section{WMH pair-wise number and individual volume differences between 1.5T and 3T}

The relative figures for WMH pair-wise number and individual volume per WMH differences between 1.5T and 3T are shown in Table 2. This Table provides information about the number and individual volume of WMH detected only on $1.5 \mathrm{~T}$ or only on 3T, and those detected in common on both scanners. A significantly greater number of WMH was found on 3T compared to $1.5 \mathrm{~T}(p<0.001)$. WMH individual volume was also significantly higher on 3T $(p<0.001)$, compared to 1.5T. A scatter plot of WMH volumes on $1.5 \mathrm{~T}$ and $3 \mathrm{~T}$ is shown in Figure 1.

Table 2. Number and individual volume of white-matter hyperintensities in healthy individuals.

\begin{tabular}{llll} 
& \multirow{2}{*}{ Number of WMH } & \multicolumn{2}{l}{ Individual volume of WMH in $\mathbf{~ m m}^{\mathbf{3}}$} \\
\cline { 3 - 4 } & & mean (SD) & median (min-max) \\
\hline $\begin{array}{l}\text { Detected only on 1.5T } \\
\text { Detected only on 3T }\end{array}$ & 25 & $29.9(32.5)$ & $36.9(2.6-137.1)$ \\
Detected on both scanners (in common) & & $79.6(131.2)$ & \\
1.5T & 72 & & $69.8(5.2-332.4)$ \\
3T & & $88.2(68.7)$ & $152.9(5.2-1017.8)$ \\
\hline
\end{tabular}

Note. WMH: White matter hyperintensities, SD: standard deviation, min: minimum value, max: maximum value, T: Tesla. 
Figure 1. Scatter plot showing volume of WMH on $1.5 \mathrm{~T}$ vs $3 \mathrm{~T}$. Values are jittered by $1 \%$ to emphasize density

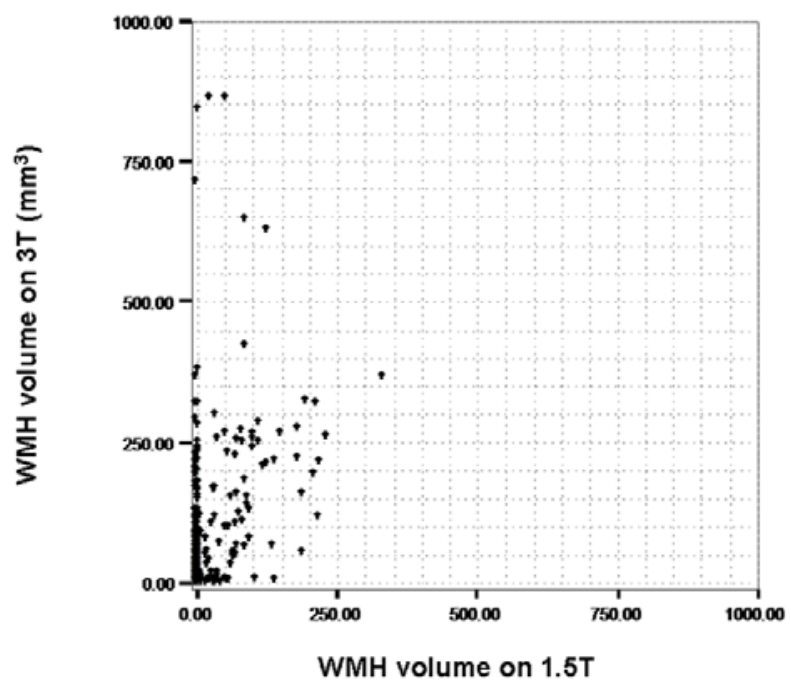

Since the individual WMH volume differed between the 1.5T and 3T scans, the maximum individual volume on either scan was considered as the best estimate of true WMH individual volume. Individual WMH volume was a significant predictor $(p<0.001)$ of whether WMH would be depicted on 1.5T, with smaller WMH being more likely to be missed on 1.5T. For every $100 \mathrm{~mm} 3$ decrease in individual volume, the odds of being missed on $1.5 \mathrm{~T}$ increased by $34 \%$.

\section{Voxel-wise size WMH differences between 1.5 $\mathrm{T}$ and $3 \mathrm{~T}$}

The voxel-wise analysis of WMH distribution showed significant differences in the prevalence of WMH in numerous areas of the brain. Most notably, the prevalence of WMH was significantly higher on 3T than on 1.5T in the occipital lobes and in the frontal horns (see Figure 2).

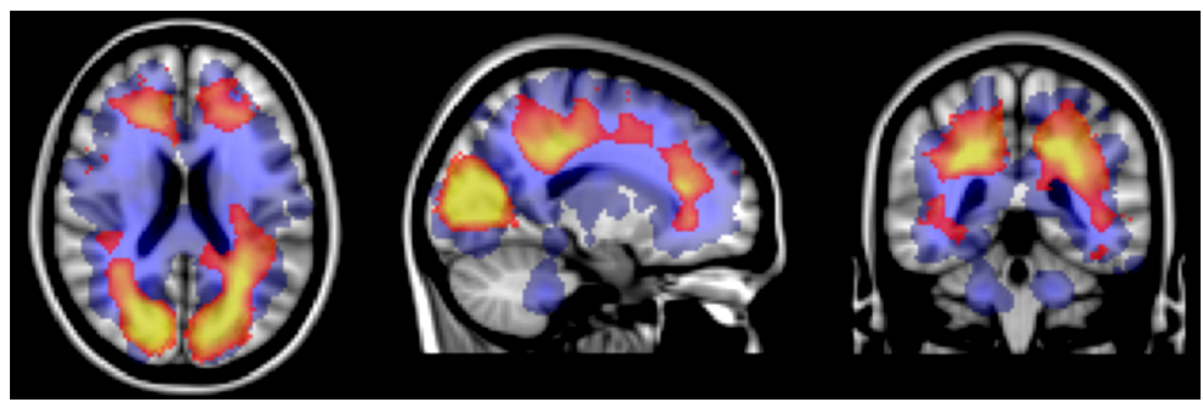

Figure 2. Voxel-wise statistical comparison of WMH prevalence between 1.5T and 3T scans. Red-yellow areas represent significant ( $p$ $<0.05$ corrected, TFCE technique) differences with a scale of 0-5 representing voxel-wise T-values. Areas containing any WMH at all are overlaid with semi-transparent blue to facilitate interpretation (i.e., gray areas contained no WMH for any subjects at any strength, so no statistical comparison can be drawn there).

\section{A posteriori non-concordant unblinded analysis}

Of all WMH, 173 were originally found only on 3T images; of these, 45 (26\%) were later also assigned to the 1.5T scans based on side-by-side comparison. In contrast, of $25 \mathrm{WMH}$ originally found only on the $1.5 \mathrm{~T}$ image, all of them (100\%) were later assigned to $3 \mathrm{~T}$ scans based on a side-by-side comparison of the scans. The number of WMH assigned a posteriori to the 3T scanner was significantly higher $(p<0.001$, see Figure 3$)$. 


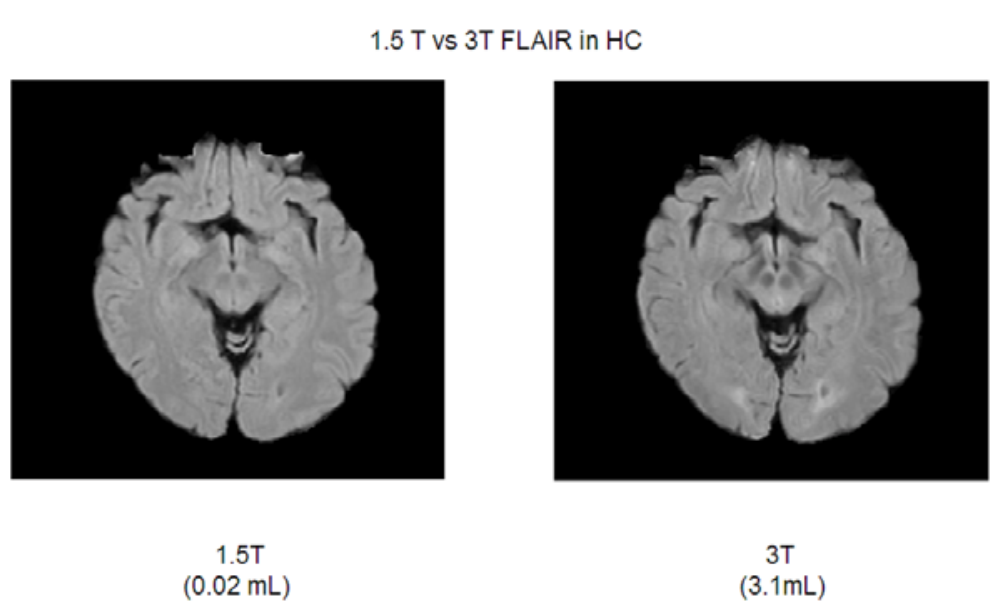

Figure 3. Examples of comparison of WMH in HC between 1.5T FLAIR (on the left) and 3T FLAIR (on the right). At higher field strength, WMH appear to be more evident.

\section{Discussion}

Our study showed that the use of higher magnetic field strength in HI resulted in the identification of more WMH regarding both morphological and spatial characteristics.

Presence of WMH reflects damage in the WM with increased water content and degeneration of macromolecular structures, resulting in a change to the tissue's relaxation times and, as a consequence of this, they appear as high intensity signals on T2-weighted scans such as FLAIR MRI sequence. Still, despite a uniform appearance on MRI, radiologicpathologic studies have demonstrated that WMH are histologically heterogenous ${ }^{[3,34]}$. The most important and consistent risk factor for WMH appeared to be age ${ }^{[35]}$, while other suggested risk factors included elevated systolic blood pressure ${ }^{[4]}$, aortic atherosclerosis ${ }^{[36]}$, atrial fibrillation ${ }^{[37]}$ and heart failure ${ }^{[38]}$.

An increased interest in detecting WMH is to some extent a product of technological improvement. In the early seventies, the use of computed tomography (CT) detected hypointense areas in the brain corresponding to WM changes (termed "Binsvanger's disease") which were previously only detected post-mortem. The introduction of MRI led to the detection of T2 hyperintense lesions that were undetectable using CT scans in both diseased and asymptomatic subjects ${ }^{\text {[39] }}$. Recently, several MRI studies have focused on unselected healthy populations and have concluded that WMH are common incidental findings not only in the brains of elderly subjects ${ }^{[1,3,40]}$ but also in the middle-aged ${ }^{[6,41]}$ and the young $\mathrm{HI}^{[42]}$.

Due to the impact of the increasing availability of high-field strength, it has now become very important to understand whether and to what extent the use of these higher fields can influence the detection of WMH in HI. Higher strength fields have been shown to detect more WMH in diseased individuals such as MS patients ${ }^{[11-15]}$ and recently in $\mathrm{HI}{ }^{[19]}$ concluding that discrete and diffuse parenchymal brain white matter FLAIR hyperintensities are more common and prominent at 3T than at $1.5 \mathrm{~T}$ in $\mathrm{HI}$.

To the best of our knowledge, this is the first study to assess morphological and spatial WMH differences in a large HI population using both $1.5 \mathrm{~T}$ and $3 \mathrm{~T}$ by applying fully automated paired and voxel-wise quantitative comparison methods. We found that for pair-wise per WMH number and individual volume analyses, based on all WMH detected either on 1.5T or 3T, 3T showed a 54.7\% increase in the number of WMH and 4 times higher WMH individual volume compared to 1.5T. The voxel-wise analysis demonstrated that the increased detection of WMH was not spatially homogeneous - i.e., it was more likely to see increased WMH in specific areas such as the frontal and occipital periventricular regions, possibly as 
these areas are notably more susceptible to vascular hypoperfusion ${ }^{[43]}$. Our findings thus confirm that 3T field strength offers the opportunity to examine WM hyperintensities in greater detail than $1.5 \mathrm{~T}$ even in a clinically healthy and young population.

Some of the robust aspects of this study are the relatively large size of the population, the careful screening of our healthy population, the short period of time between scans, the effort made to establish identical scanning conditions between both scanners, the randomized scanner order per subject and the blinded analysis of the MRI scans. In comparison to previous studies, this is the first to evaluate paired differences in individual volume and distribution of brain WMH detected on 1.5T and 3T scanners in a large population of HI. The automated procedures that were applied to both lesion-wise and voxel-wise comparisons assured highly reliable results.

Our findings have several implications for clinical routine. These WMH might not necessarily be interpreted as pathologic findings as they may be seen in a healthy highly screened population, suggesting that they are related to neuronal degenerative processes and detected only on 3T. As an alternative hypothesis, these WMH detected on 3T might indicate preclinical signs of future disabling, but possibly reversible and treatable, pathology and, therefore, should prompt screening for stroke and dementia risk factors ${ }^{[44]}$. Whether higher WMH number and volume on 3T may actually reflect "real pathology" is thus at the present time still unclear and goes beyond our study, as additional future studies are warranted—including larger cohorts and preferably of longitudinal design— to investigate the pathological nature of these findings. However,

In conclusion, this lesion- and voxel-wise comparison study between 1.5T and 3T scanners in HC provides important information regarding morphological and spatial differences between the two scanner field strengths. High field MRI systems (3T) facilitate the detection of more brain MRI WMH, and the increased detection may not be spatially homogeneous - i.e., it may be more likely to see increased signal abnormalities in specific areas such as the occipital lobes. Such hyperintensities do not necessarily reflect brain pathology or pre-pathology, as future studies are warranted in order to clarify this aspect. However, our findings should be taken into account in all clinical trials based on lesion segmentation on a 3T. Furthermore, they might suggest the need of universally accepted guidelines for lesions' segmentation on a 3T, especially when executed by different operators and/or clinical centers.

\section{Acknowledgements}

This study was performed as part of a research collaboration agreement among General Electric Company, The State University of New York at Buffalo, Kaleida Health Systems and University Neurology, Inc. Dr. Zivadinov served as PI on that agreement. The authors have no financial relationship to disclose. Dr. Carol Di Perri was supported by the Dr. Larry D. Jacobs Jog-for-the-Jake Fellowship. Dr. Turi O. Dalaker was supported by the Dr. Larry D. Jacobs Jog-for -the -Jake Fellowship and The Leiv Eiriksson programme from the Research Council of Norway (grant\# 186966). We also thank Eve Salczynski for technical support in the preparation of this manuscript.

\section{References}

[1] Enzinger C., Smith S., Fazekas F., Drevin G, Ropele S, Nicholss T. et al. Lesion probability maps of white matter hyperintensities in elderly individuals: results of the Austrian stroke prevention study. J Neurol. 2006; 253: 1064-70. PMid:16607471 http://dx.doi.org/10.1007/s00415-006-0164-5

[2] Fazekas F. Magnetic resonance signal abnormalities in asymptomatic individuals: their incidence and functional correlates. Eur Neurol. 1989; 29: 164-8. PMid:2731564 http://dx.doi.org/10.1159/000116401

[3] Hopkins RO, Beck CJ, Burnett DL, Weaver LK, Victoroff J, Bigler ED. Prevalence of white matter hyperintensities in a young healthy population. J Neuroimaging. 2006; 16: 243-51. PMid:16808826 http://dx.doi.org/10.1111/j.1552-6569.2006.00047.x

[4] Longstreth WT, Jr. Manolio TA, Arnold A, Burke GL, Bryan N, Jungreis CA. Clinical correlates of white matter findings on cranial magnetic resonance imaging of 3301 elderly people. The Cardiovascular Health Study. Stroke. 1996; 27: $1274-82$. PMid:8711786 http://dx.doi.org/10.1161/01.STR.27.8.1274 
[5] Wen W and Sachdev P. The topography of white matter hyperintensities on brain MRI in healthy 60- to 64-year-old individuals. Neuroimage. 2004; 22: 144-54. PMid:15110004 http://dx.doi.org/10.1016/j.neuroimage.2003.12.027

[6] Wen W, Sachdev PS, Li JJ, Chen X, Anstey KJ. White matter hyperintensities in the forties: their prevalence and topography in an epidemiological sample aged 44-48. Hum Brain Mapp. 2009; 30: 1155-67. PMid:18465744 http://dx.doi.org/10.1002/hbm.20586

[7] Gunning-Dixon FM and Raz N. The cognitive correlates of white matter abnormalities in normal aging: a quantitative review. Neuropsychology. 2000; 14: 224-32. PMid:10791862 http://dx.doi.org/10.1037/0894-4105.14.2.224

[8] Sachdev PS, Wen W, Christensen H, Jorm AF. White matter hyperintensities are related to physical disability and poor motor function. J Neurol Neurosurg Psychiatry. 2005; 76: 362-7. PMid:15716527 http://dx.doi.org/10.1136/jnnp.2004.042945

[9] Sakakibara R, Hattori T, Uchiyama T, Yamanishi T. Urinary function in elderly people with and without leukoaraiosis: relation to cognitive and gait function. J Neurol Neurosurg Psychiatry. 1999; 67: 658-60. PMid:10519875 http://dx.doi.org/10.1136/jnnp.67.5.658

[10] Steffens DC, Bosworth HB, Provenzale J M, Berger K. Subcortical white matter lesions and functional impairment in geriatric depression. Depress Anxiety. 2002; 15: 23-8. PMid:11816049 http://dx.doi.org/10.1002/da.1081

[11] Keiper MD, Grossman RI, Hirsch, JA, Bolinger L, Ott IL, Mannon LJ, et al. MR identification of white matter abnormalities in multiple sclerosis: a comparison between 1.5 T and 4 T. AJNR Am J Neuroradiol. 1998; 19: 1489-93. PMid:9763383

[12] Sicotte NL, Voskuhl RR, Bouvier S, Klutch R, Cohen MS, Mazziotta JC. Comparison of multiple sclerosis lesions at 1.5 and 3.0 Tesla. Invest Radiol. 2003; 38: 423-7. PMid:12821856 http://dx.doi.org/10.1097/01.RLI.0000065426.07178.f1

[13] Lee DH, Vellet AD, Eliasziw M, Klutch R, Cohen MS, Mazziotta JC. MR imaging field strength: prospective evaluation of the diagnostic accuracy of MR for diagnosis of multiple sclerosis at 0.5 and 1.5 T. Radiology. 1995; 194: 257-62. PMid:7997564

[14] Fischbach $\mathrm{F}$ and Bruhn $\mathrm{H}$. Assessment of in vivo 1H magnetic resonance spectroscopy in the liver: a review. Liver Int. 2008; 28: 297-307. PMid:18290772 http://dx.doi.org/10.1111/j.1478-3231.2007.01647.x

[15] Di Perri C, Dwyer M G, Wack DS, Cox JL, Hashmi K, Saluste E et al. Signal abnormalities on 1.5 and 3 Tesla brain MRI in multiple sclerosis patients and healthy controls. A morphological and spatial quantitative comparison study. Neuroimage. 2009; 47: 1352-62. PMid:19371784 http://dx.doi.org/10.1016/j.neuroimage.2009.04.019

[16] Wattjes MP, Harzheim M, Kuhl CK, Gieseke J, Schmidt S, Klotz L et al. Does high-field MR imaging have an influence on the classification of patients with clinically isolated syndromes according to current diagnostic mr imaging criteria for multiple sclerosis? AJNR Am J Neuroradiol. 2006; 27: 1794-8. PMid:16971638

[17] Zijlmans M, de Kort GA, Witkamp T D, Huiskamp GM, Seppenwoolde JH, van Huffelen AC, et al. 3T versus 1.5T phased-array MRI in the presurgical work-up of patients with partial epilepsy of uncertain focus. J Magn Reson Imaging. 2009 ; 30: $256-62$. PMid:19629993 http://dx.doi.org/10.1002/jmri.21811

[18] Nandigam RN, Viswanathan A, Delgado P, Skehan ME, Smith EE, Rosand J, et al. MR imaging detection of cerebral microbleeds: effect of susceptibility-weighted imaging, section thickness, and field strength. AJNR Am J Neuroradiol. 2009; 30: 338-43. PMid:19001544 http://dx.doi.org/10.3174/ajnr.A1355

[19] Neema M, Guss ZD, Stankiewicz JM, Arora A, Healy BC, Bakshi R. Normal findings on brain fluid-attenuated inversion recovery MR images at 3T. AJNR Am J Neuroradiol. 2009; 30: 911-6. PMid:19369605 http://dx.doi.org/10.3174/ajnr.A1514

[20] DeCarli C, Fletcher E, Ramey V, Harvey D, Jagust WJ. Anatomical mapping of white matter hyperintensities (WMH): exploring the relationships between periventricular WMH, deep WMH, and total WMH burden. Stroke. 2005; 36: 50-5. PMid:15576652 http://dx.doi.org/10.1161/01.STR.0000150668.58689.f2

[21] Di Perri C, Battaglini M, Stromillo ML, Bartolozzi ML, Guidi L, Federico A et al. Voxel-based assessment of differences in damage and distribution of white matter lesions between patients with primary progressive and relapsing-remitting multiple sclerosis. Arch Neurol. 2008; 65: 236-43. PMid:18268194 http://dx.doi.org/10.1001/archneurol.2007.51

[22] Ghassemi R, Antel SB, Narayanan S, Francis SJ, Bar-Or A, Sadovnick AD. Lesion distribution in children with clinically isolated syndromes. Ann Neurol. 2008; 63: 401-5. PMid:18306242 http://dx.doi.org/10.1002/ana.21322

[23] Folstein MF, Folstein SE and McHugh PR. "Mini-mental state". A practical method for grading the cognitive state of patients for the clinician. J Psychiatr Res. 1975; 12: 189-98. http://dx.doi.org/10.1016/0022-3956(75)90026-6

[24] Smith A. Symbol digit modalities test: Manual. Los Angeles: Western Psychological Services. 1982.

[25] Zivadinov R, Rudick RA, De Masi R, Nasuelli D, Ukmar M, Pozzi-Mucelli RS et al. Effects of IV methylprednisolone on brain atrophy in relapsing-remitting MS. Neurology. 2001; 57: 1239-47. PMid:11591843 http://dx.doi.org/10.1212/WNL.57.7.1239

[26] Ylikoski A, Erkinjuntti T, Raininko R, Sarna S, Sulkava R, Tilvis R. White matter hyperintensities on MRI in the neurologically nondiseased elderly. Analysis of cohorts of consecutive subjects aged 55 to 85 years living at home. Stroke. 1995; 26: $1171-7$. PMid:7604409 http://dx.doi.org/10.1161/01.STR.26.7.1171 
[27] van Swieten JC, van den Hout JH, van Ketel BA, Hijdra A, Wokke JH, van Gijn J. Periventricular lesions in the white matter on magnetic resonance imaging in the elderly. A morphometric correlation with arteriolosclerosis and dilated perivascular spaces. Brain. 1991; 114 (Pt 2): 761-74. PMid:2043948 http://dx.doi.org/10.1093/brain/114.2.761

[28] Vermeer SE, Hollander M, van Dijk EJ, Hijdra A, Wokke JH, van Gijn J. Silent brain infarcts and white matter lesions increase stroke risk in the general population: the Rotterdam Scan Study. Stroke. 2003; 34: 1126-9. PMid:12690219 http://dx.doi.org/10.1161/01.STR.0000068408.82115.D2

[29] Smith SM, Jenkinson M, Woolrich MW, Beckmann CF, Behrens TE, Johansen-Berg H et al. Advances in functional and structural MR image analysis and implementation as FSL. Neuroimage. 2004; 23 Suppl 1: S208-19. PMid:15501092 http://dx.doi.org/10.1016/j.neuroimage.2004.07.051

[30] Ashburner J and Friston KJ. Why voxel-based morphometry should be used. Neuroimage. 2001; 14: 1238-43. PMid:11707080 http://dx.doi.org/10.1006/nimg.2001.0961

[31] Nichols TE and Holmes AP. Nonparametric permutation tests for functional neuroimaging: a primer with examples. Hum Brain Mapp. 2002; 15: 1-25. PMid:11747097 http://dx.doi.org/10.1002/hbm.1058

[32] Smith SM and Nichols TE. Threshold-free cluster enhancement: addressing problems of smoothing, threshold dependence and localisation in cluster inference. Neuroimage. 2009; 44: 83-98. PMid:18501637 http://dx.doi.org/10.1016/j.neuroimage.2008.03.061

[33] Spilt A, Goekoop R, Westendorp RG, Blauw GJ, de Craen AJ, van Buchem MA. Not all age-related white matter hyperintensities are the same: a magnetization transfer imaging study. AJNR Am J Neuroradiol. 2006; 27: 1964-8. PMid:17032876

[34] Fazekas F, Kleinert R, Offenbacher H, Schmidt R, Kleinert G, Payer F, et al. Pathologic correlates of incidental MRI white matter signal hyperintensities. Neurology. 1993; 43: 1683-9. PMid:8414012 http://dx.doi.org/10.1212/WNL.43.9.1683

[35] de Leeuw FE, de Groot JC, Achten E, Oudkerk M, Ramos LM, Heijboer R, et al. Prevalence of cerebral white matter lesions in elderly people: a population based magnetic resonance imaging study. The Rotterdam Scan Study. J Neurol Neurosurg Psychiatry. 2001; 70: 9-14. PMid:11118240 http://dx.doi.org/10.1136/jnnp.70.1.9

[36] de Leeuw FE, De Groot JC, Oudkerk M, Witteman JC, Hofman A, van Gijn J, et al. Aortic atherosclerosis at middle age predicts cerebral white matter lesions in the elderly. Stroke. 2000; 31: 425-9. PMid:10657417 http://dx.doi.org/10.1161/01.STR.31.2.425

[37] de Leeuw FE, de Groot JC, Oudkerk, M, Kors JA, Hofman A, van Gijn J et al. Atrial fibrillation and the risk of cerebral white matter lesions. Neurology. 2000; 54: 1795-801. PMid:10802786 http://dx.doi.org/10.1212/WNL.54.9.1795

[38] Vogels RL, Oosterman JM, van Harten B, Gouw AA, Schroeder-Tanka JM, Scheltens P et al. Neuroimaging and correlates of cognitive function among patients with heart failure. Dement Geriatr Cogn Disord. 2007; 24: 418-23. PMid:17938570 http://dx.doi.org/10.1159/000109811

[39] Pantoni L. and Garcia JH. The significance of cerebral white matter abnormalities 100 years after Binswanger's report. A review. Stroke. 1995; 26: 1293-301. PMid:7604429 http://dx.doi.org/10.1161/01.STR.26.7.1293

[40] Qu H, Nishimaru K, Utsunomiya H, Une H. [Neuroradiological study of cerebral microlesions (2): changes in elderly]. No To Shinkei. 2003; 55: 675-82. PMid:13677301

[41] Sachdev P, Chen,X and Wen W. White matter hyperintensities in mid-adult life. Curr Opin Psychiatry. 2008 ; $21: 268-74$. PMid:18382226 http://dx.doi.org/10.1097/YCO.0b013e3282f945d5

[42] Kumra S, Ashtari M, Anderson B, Cervellione KL, Kan L. Ethical and practical considerations in the management of incidental findings in pediatric MRI studies. J Am Acad Child Adolesc Psychiatry. 2006; 45: 1000-6. PMid:16865043 http://dx.doi.org/10.1097/01.chi.0000222786.49477.a8

[43] Kawamura J, Meyer JS, Terayama Y, Weathers S. Leukoaraiosis correlates with cerebral hypoperfusion in vascular dementia. Stroke. 1991; 22: 609-14. PMid:2028490 http://dx.doi.org/10.1161/01.STR.22.5.609 